\title{
Applicability of the Fourier Expansion Method of Separation of Variables in the Linear Discrete-Continuous Systems with Distributional Coefficients
}

\begin{abstract}
S. KASPRZYK
Katedra Mechaniki i Wibroakustyki, AGH — UST, al. A. Mickiewicza 30, 30-059 Kraków, Poland

The analytical examination of mechanical systems in the aspect of their vibro-isolation can be limited to the construction of a computational system. The analytical description of the adopted appropriate computational model may be executed with the help of a set of differential equations of the second order, differential equations with partial derivatives or of both types at the same time. The latest description is associated with the so-called discrete-continuous systems. It is the most convenient to analyze the vibrations of the linear discrete-continuous systems in the class of functions generalized with the Fourier method of separation of variables. Until now it was possible to execute only for a small set of parameters of the system's structure. In the work the author presents a computational model that covers all the structural parameters of the system.
\end{abstract}

PACS: 46.70.De, 46.40.-f

\section{Introduction}

The results of the analysis of vibrations depend on the adopted computational system. The computational system should be characterized by the following features:

1. it should take into account the essential features of the physical system in the aspect of vibrations, 2. it should be simple in its mathematical description, which is essential for the course of the analysis, 3 . in the case of the mathematical description dependent upon two or more independent variables it is favourable if the variables can be separated.

Such features are possessed by the discrete-continuous systems considered in the class of generalized functions. Fourier's method brings positive results only for a small set of the structure parameters. In this work the presented method of separations of variables is applicable for all the parameters of the structure.

Vibrations of mechanical systems with distributed parameters are described by functions dependent upon two variables: the spatial one and the temporal one. An analysis of vibrations of this kind of systems can be well conducted with the Fourier method of separation of variables. Typical systems with distributed parameters for which it is appropriate to conduct an analysis with the method of separation of variables include the longitudinal vibrations of a rod, transverse vibrations of a beam, torsional vibrations of a shaft and vibrations of discrete-continuous systems.

A typical discrete-continuous system is presented in Fig. 1.

Model in Fig. 1 is equivalent of general physical discrete-continuous system. Description of the model: $O B$ - Euler's beam, freely supported at its ends, whose length $l>0,0<E J$ bending rigidity of the beam,

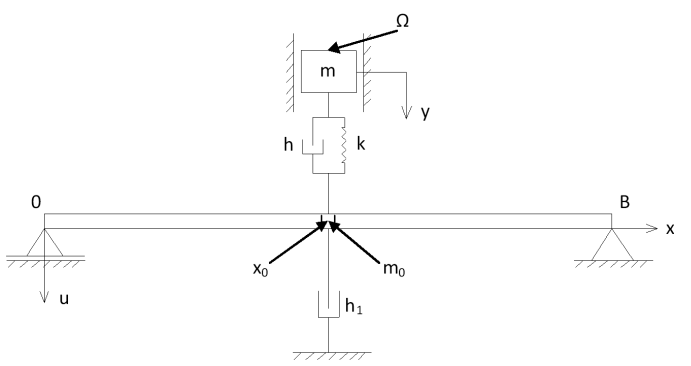

Fig. 1. A model of a discrete-continuous system.

$0<\rho F-$ specific weight, $0<m_{0}$ - mass concentrated around the point $x_{0}$ of the beam, in which massless constraints with parameters $0 \leq h, 0 \leq k$ are connected pointwise, $0 \leq \alpha_{0}$ - coefficient of structural damping of the beam; $0 \leq m-$ mass of the material point $\Omega ; y(t)$ function describing the deviation from the balanced position of $\Omega ; u(x, t)$ - function describing deviation from the balanced position of the beam particles; $f_{1}(x, t)$ and $f_{2}(t)$ - functions describing external forces acting on the system presented in Fig. 1.

Vibrations of the system presented in Fig. 1 are described by the following system of equations [1-4]:

$$
\begin{aligned}
& E I \frac{\partial^{4} u}{\partial x^{4}}+I \alpha_{0} \frac{\partial^{5} u}{\partial x^{4} \partial t}+\left(m_{0} \delta_{x_{0}}+\rho F\right) \frac{\partial^{2} u}{\partial t^{2}} \\
& +\left[k\left(u\left(x_{0}, t\right)-y(t)\right)+h\left(\frac{\partial u\left(x_{0}, t\right)}{\partial t}-\dot{y}(t)\right)\right] \delta_{x_{0}} \\
& \quad+h_{1} \frac{\partial u\left(x_{0}, t\right)}{\partial t} \delta_{x_{0}}=f_{1}(x, t) \\
& m \ddot{y}+h\left(\dot{y}(t)-\frac{\partial u\left(x_{0}, t\right)}{\partial t}\right) \\
& \quad+k\left[y(t)-u\left(x_{0}, t\right)\right]=f_{2}(t)
\end{aligned}
$$

with the boundary conditions 


$$
u(0, t)=u(l, t)=0, \quad \frac{\partial^{2} u(0, t)}{\partial x^{2}}=\frac{\partial^{2} u(l, t)}{\partial x^{2}}=0
$$

and the initial conditions

$$
u(0, t)=\varphi_{0}(x), \quad \frac{\partial u(x, 0)}{\partial t}=\varphi_{1}(x)
$$

where $\delta_{x_{0}}$ is the Dirac delta function concentrated in $x_{0}$.

The system (1), (2), (3) is called the initial-boundary problem.

All equalities are understood in the distributive sense; differential coefficients are also understood in the distributive sense $[1,5]$. The mass $m_{0}$ in $x_{0}$ represents mass increase in the neighbourhood of $x_{0}$ caused by the connection of the constraints with the beam, while $h_{1}$ is the change of damping in the neighbourhood of $x_{0}$.

\section{Explanation of the subject under study}

The hitherto approach to the solution of the problem defined in Eqs. (1)-(3) with the method of separation of variables is successful for the cases

$$
\begin{aligned}
& \alpha_{0}=0, \quad h=0, \quad h_{1}=0, \\
& \frac{\alpha_{0}}{E}=\frac{h}{k}, \quad h_{1}=0,
\end{aligned}
$$

when we assume

$$
u(x, t)=X(x) T(t), \quad y(t)=A T(t) .
$$

For the relation

$$
\frac{\alpha_{0}}{E} \neq \frac{h}{k}, \quad h_{1} \neq 0
$$

we shall use the modified method, that is, we shall assume

$$
u(x, t)=X(x) T(t), \quad y(t)=A S(t) .
$$

The basic goal of the work is to offer a new approach to the method of separation of variables for the solution of Eqs. (1), (2) for (6) as well as a model of the solution of the problem defined in Eqs. (1)-(3).

In order to achieve the main goal of the study we shall neglect the external forces acting on the system.

To facilitate understanding of the idea of realization of the main goal, let us quote own problems and the equation of the function $T(t)$ for (3) and (4). In (3) and (4) we apply the standard procedure while (6) requires a proof.

The results of the solution of the problem defined in Eqs. (1), (2) for (3), (4) and (6) are included in the theorem:

\section{Theorem}

From (1), (2) and (5) for (4) it follows that:

$$
\begin{aligned}
& X^{I V}-\lambda^{4} X=\left(\frac{k}{\omega_{0}^{2}-\omega^{2}}+m_{0}\right) \frac{\omega^{2}}{E I} X\left(x_{0}\right) \delta_{x_{0}}, \\
& \omega_{0}^{2}=\frac{k}{m}, \quad \lambda^{4}=\frac{\rho F \omega^{2}}{E I},
\end{aligned}
$$

$$
\begin{aligned}
& \frac{X\left(x_{0}\right)}{A}=\frac{\omega_{0}^{2}-\omega^{2}}{\omega_{0}^{2}}, \quad \omega_{o} \neq \omega, \\
& X(0)=X(l)=0, \quad X^{\prime \prime}(0)=X^{\prime \prime}(l)=0, \\
& \ddot{T}+\omega^{2} T=0 .
\end{aligned}
$$

Equation (9) is obtained from

$$
\begin{aligned}
& \frac{E I X^{I V}+k\left(X\left(x_{0}\right)-A\right) \delta_{x_{0}}}{\left(m_{0} \delta_{x_{0}}+\rho F\right) X}=\omega^{2}, \\
& \left(m_{0} \delta_{x_{0}}+\rho F\right) X(x) \neq 0, \quad x \in\langle 0, l\rangle .
\end{aligned}
$$

Applying (1) and (13) we obtain (10).

By analogy to (6), for (5) we obtain eigenproblem (9), (10), (11) and

$$
\ddot{T}+\frac{\alpha_{0}}{E} \omega^{2} \dot{T}+\omega^{2} T=0 .
$$

From (1), (2) and (8), taking into account

$$
h+h_{1}=k \frac{\alpha_{0}}{E}
$$

we obtain eigenproblem (9), (10) and for $x \in(\langle 0, l\rangle-$ $\left.\left\{x_{0}\right\}\right)$

$$
\ddot{T}+\frac{\alpha_{0}}{E} \omega^{2} \dot{T}+\omega^{2} T=0,
$$

while for $x=x_{0}$

$$
\begin{aligned}
& \ddot{T}+\left(\omega^{2}+\frac{k \omega_{0}^{2}}{m_{0}\left(\omega_{0}^{2}-\omega^{2}\right)}\right)\left(\frac{\alpha_{0}}{E} \dot{T}+T\right) \\
& -\frac{k \omega_{0}^{2}}{m_{0}\left(\omega_{0}^{2}-\omega^{2}\right)}\left(\frac{h}{k} \dot{S}+S\right)=0, \\
& \ddot{S}+2 \alpha \dot{S}+\omega_{0}^{2} S-\left(1-\frac{\omega^{2}}{\omega_{0}^{2}}\right)\left(2 \alpha \dot{T}+\omega_{0}^{2} T\right)=0, \\
& \quad 2 \alpha=\frac{h}{m} .
\end{aligned}
$$

Further in the work we shall show that the denominator of (13) is different than zero. Equation (9) is valid for $\omega_{0} \neq \omega$. It issues from the Theorem that the new idea of applicability expansion of the method of variables separation consists in invariance of eigenproblems.

In order to demonstrate (16), (17) and identity of (6) with (4), we must show

\section{Lemma}

A : $\quad \frac{X\left(x_{0}\right)}{A}=\frac{\omega_{0}^{2}-\omega^{2}}{\omega_{0}^{2}}, \quad$ see $(10)$;

$\mathrm{T}: \frac{k A \delta_{x_{0}}}{\left(m_{0} \delta_{x_{0}}+\rho F\right) X}$ $=\left\{\begin{array}{cc}\frac{k A}{m_{0} X\left(x_{0}\right)} & \text { for } x=x_{0}, \quad m_{0}>0, \quad \rho F>0, \\ 0 & \text { for } x \neq x_{0} .\end{array}\right.$

Proof: let us set 


$$
\begin{aligned}
& \frac{k A \delta_{x_{0}}}{\left(m_{0} \delta_{x_{0}}+\rho F\right) X(x)}=a, \quad x=x_{0}, \\
& \frac{k A \delta_{x_{0}}}{\left(m_{0} \delta_{x_{0}}+\rho F\right) X(x)}=b, \quad x \neq x_{0}, \\
& H\left(x_{0}, \varepsilon\right)=H\left(x_{0}-\varepsilon\right)-H\left(x_{0}+\varepsilon\right), \quad \varepsilon>0, \\
& \tilde{H}\left(x_{0}, \varepsilon\right)=H(0)-H(l)-H\left(x_{0}, \varepsilon\right), \\
& H(\xi)=1, \quad x>\xi, \quad H(\xi)=0, \quad \xi<0, \quad H(\xi)=\frac{1}{2} .
\end{aligned}
$$

We multiply both sides of (18) by the denominator, then we multiply $H\left(x_{0}, \varepsilon\right)$ and $\varphi(x)$, and integrate, obtaining

$$
\begin{aligned}
& k A \varphi\left(x_{0}\right)=a m_{0} X\left(x_{0}\right) \varphi\left(x_{0}\right) \\
& +a \rho F \int_{\mathbb{R}} X(x) H\left(x_{0}, \varepsilon\right) \varphi\left(x_{0}\right) \mathrm{d} x .
\end{aligned}
$$

$\varphi \in D$ is a set of trial functions [5].Moving from $\varepsilon \rightarrow 0$ we have

$$
k A \varphi\left(x_{0}\right)=a m_{0} X\left(x_{0}\right) \varphi\left(x_{0}\right)
$$

or

$\left[k A-a m_{0} X\left(x_{0}\right)\right] \varphi\left(x_{0}\right)=0$ for each $\varphi$.

From the above and the assumption of the Lemma we get

$$
a=\frac{k A}{m_{0} X\left(x_{0}\right)} .
$$

Similarly to (18) we calculate (19), but instead of $H\left(x_{0}, \varepsilon\right)$ we use $\tilde{H}\left(x_{0}, \varepsilon\right)$, obtaining

$$
0=b \int_{\mathbb{R}} \rho F X(x) \tilde{H}\left(x_{0}, \varepsilon\right) \varphi(x) \mathrm{d} x
$$

for every $\varphi \in D$. Hence $b=0$ because the integral is different than zero. In a similar way to that in (18) and (19) we find that

$$
m_{0} X\left(x_{0}\right) \delta_{x_{0}}+\rho F X(x) \neq 0 \quad \text { for } \quad x=x_{0}
$$

and for $x \in\left(\langle 0, l\rangle-\left\{x_{0}\right\}\right)$.

Proof for (6):

Inserting Eq. (7) into the first equation of (1) we obtain

$$
\begin{aligned}
& E I\left(T+\frac{\alpha_{0}}{E} \dot{T}\right)+\left(m_{0} \delta_{x_{0}}+\rho F\right) X \ddot{T}+k X\left(x_{0}\right) \\
& \quad \times\left(T+\frac{h+h_{1}}{k} \dot{T}\right) \delta_{x_{0}}-k A\left(S+\frac{h}{k} \dot{S}\right) \delta_{x_{0}}=0 .
\end{aligned}
$$

From (20) and (15) we have

$$
\begin{gathered}
-\frac{E I X^{I V}+k X\left(x_{0}\right) \delta_{x_{0}}}{\left(m_{0} \delta_{x_{0}}+\rho F\right) X(x)}=\frac{\ddot{T}}{T+\frac{\alpha_{0}}{E} \dot{T}} \\
-\frac{k A\left(S+\frac{h}{k} \dot{S}\right) \delta_{x_{0}}}{\left(m_{0} \delta_{x_{0}}+\rho F\right) X\left(T+\frac{\alpha_{0}}{E} \dot{T}\right)} .
\end{gathered}
$$

Adding the formula

$$
k A \delta_{x_{0}} /\left(m_{0} \delta_{x_{0}}+\rho F\right) X
$$

to both sides of (21), we obtain

$$
\begin{gathered}
-\frac{E I X^{I V}+k\left[X\left(x_{0}\right)-A\right] \delta_{x_{0}}}{\left(m_{0} \delta_{x_{0}}+\rho F\right) X}=\frac{\ddot{T}}{T+\frac{\alpha_{0}}{E} \dot{T}} \\
+\frac{k A \delta_{x_{0}}}{\left(m_{0} \delta_{x_{0}}+\rho F\right) X}\left(1-\frac{S+\frac{h}{k} \dot{S}}{T+\frac{\alpha_{0}}{E} \dot{T}}\right) .
\end{gathered}
$$

From (22) and the Lemma we get

$$
\begin{gathered}
-\frac{E I X^{I V}+k\left[X\left(x_{0}\right)-A\right] \delta_{x_{0}}}{\left(m_{0} \delta_{x_{0}}+\rho F\right) X}=\frac{\ddot{T}}{T+\frac{\alpha_{0}}{E} \dot{T}} \\
+\left\{\begin{array}{c}
\frac{k A}{m_{0} X \alpha_{0}} \frac{T+\frac{\alpha_{0}}{E} \dot{T}-\left(S+\frac{h}{k} \dot{S}\right)}{T+\frac{\alpha_{0}}{E} \dot{T}}, \text { for } x=x_{0}, \\
0, \text { for } x \neq x_{0} .
\end{array}\right.
\end{gathered}
$$

By (10), the right side of (23) depends only on the variable $t$, and its left side is identical with the left side of (13). Hence, we get

$$
\begin{aligned}
& -\frac{E I X^{I V}+k\left[X\left(x_{0}\right)-A\right] \delta_{x_{0}}}{\ddot{T} \quad\left(m_{0} \delta_{x_{0}}+\rho F\right) X}=-\omega^{2}, \\
& \frac{T}{T+\frac{\alpha_{0}}{E} \dot{T}}=-\omega^{2}, \quad x \neq x_{0}, \\
& \ddot{T}+\frac{\alpha_{0}}{E} \omega^{2} \dot{T}+\omega^{2} T=0 \text { for } x \neq x_{0} \text {, } \\
& \frac{\ddot{T}^{E}}{T+\frac{\alpha_{0}}{E} \dot{T}}+k \frac{\omega_{0}^{2}}{\omega_{0}^{2}-\omega^{2}} \frac{T+\frac{\alpha_{0}}{E} \dot{T}-\left(S+\frac{h}{k} \dot{S}\right)}{T+\frac{\alpha_{0}}{E} \dot{T}}=-\omega^{2}, \\
& x=x_{0}, \\
& \ddot{T}+\left[\omega^{2}+\frac{k \omega_{0}^{2}}{m_{0}\left(\omega_{0}^{2}-\omega^{2}\right)}\right]\left(T+\frac{\alpha_{0}}{E} \dot{T}\right) \\
& -\frac{k \omega_{0}^{2}}{m_{0}\left(\omega_{0}^{2}-\omega^{2}\right)}\left(S+\frac{h}{k} \dot{S}\right)=0 \text { for } x=x_{0} \text {. }
\end{aligned}
$$

Inserting (8) to the second equation of (1), after executing simple transformations we obtain

$$
\frac{\ddot{S}+2 \alpha \dot{S}+\omega_{0}^{2} S}{2 d \dot{T}+\omega_{0}^{2} T}=\frac{X\left(x_{0}\right)}{A} \quad \text { for } \quad x=x_{0}, \quad 2 \alpha=\frac{h}{m} .
$$

By (10) and (29) it can be shown that

$$
\frac{\ddot{S}+2 \alpha \dot{S}+\omega_{0}^{2} S}{2 d \dot{T}+\omega_{0}^{2} T}=\frac{\omega_{0}^{2}-\omega^{2}}{\omega_{0}^{2}}
$$

$$
\begin{aligned}
& \ddot{S}+2 \alpha \dot{S}+\omega_{0}^{2} S-\left(1-\frac{\omega^{2}}{\omega_{0}^{2}}\right)\left(2 d \dot{T}+\omega_{0}^{2} T\right)=0, \\
& \quad x=x_{0} .
\end{aligned}
$$

Equation (24) is identical with (13) and Eqs. (28) and (30) are identical with the set (17), which means that the theorem has been proved. 

that:

Conclusions from the theorem: If, in (17), we assume

1. $\alpha_{0}=0, h=0$, we obtain $(12)$;

2. $\frac{\alpha_{0}}{E}=\frac{h}{k}$, we obtain (14).

3. On the other hand, if, in (20) we assume that $\alpha_{0}=0, h=0, k=0$, we obtain

$$
E I X^{I V} T+\left(m_{0} \delta_{x_{0}}+\rho F\right) X-h_{1} X\left(x_{0}\right) \delta_{x_{0}}=0 .
$$

Hence, from (13) and the Lemma it follows that:

$$
\begin{gathered}
-\frac{E I X^{I V}}{\left(m_{0} \delta_{x_{0}}+\rho F\right) X}=\frac{\ddot{T}}{T}+\frac{h_{1} \delta_{x_{0}}}{\left(m_{0} \delta_{x_{0}}+\rho F\right) X} \\
=\frac{\ddot{T}}{T}+\left\{\begin{array}{r}
\frac{h_{1}}{m_{0}} \frac{\dot{T}}{T}, \quad \text { for } \quad x=x_{0}, \\
0, \quad \text { for } \quad x \neq x_{0},
\end{array}\right\}=-\omega^{2} .
\end{gathered}
$$

The latest equation is equivalent

$$
\begin{aligned}
& X^{I V}-\lambda^{4} X=\frac{m_{0}}{E I} \omega^{2} \delta_{x_{0}}, \\
& \ddot{T}+\frac{h_{1}}{m_{0}} \dot{T}+\omega^{2} T=0 \text { for } x=x_{0}, \\
& \ddot{T}+\omega^{2} T=0 \text { for } x \neq x_{0} .
\end{aligned}
$$

4. Similarly as in (3), if, in (20) we assume that $\alpha_{0}=0$, and $h+h_{1}=0$, we obtain

$$
\begin{aligned}
& E I X^{I V} T+\left(m_{0} \delta_{x_{0}}+\rho F\right) X \ddot{T}+k X\left(x_{0}\right) T \delta_{x_{0}} \\
& \quad-k A\left(S+\frac{h}{k} \dot{S}\right) \delta_{x_{0}}=0 .
\end{aligned}
$$

Thus, from (10) and the Lemma, we have

$$
\begin{aligned}
- & \frac{E I X^{I V}+k X\left(x_{0}\right) \delta_{x_{0}}}{\left(m_{0} \delta_{x_{0}}+\rho F\right) X} \\
= & \frac{\ddot{T}}{T}-\left\{\begin{array}{r}
\frac{1}{T} \frac{k}{m_{0}} \frac{\omega_{0}^{2}}{\omega_{0}^{2}-\omega^{2}}\left(S+\frac{h}{k} \dot{S}\right) \text { for } x=x_{0} \cdot \\
0 \text { for } x \neq x_{0},
\end{array}\right\} \\
=-\omega^{2} . &
\end{aligned}
$$

And then

$$
\begin{aligned}
& X^{I V}-\lambda^{4} X=\frac{1}{E I}\left(m_{0} \omega^{2}-k\right) X\left(x_{0}\right) \delta_{x_{0}}, \\
& \ddot{T}+\omega^{2} T=0 \text { for } x \neq x_{0}, \\
& \ddot{T}+\omega^{2} T-\frac{k}{m_{0}} \frac{\omega_{0}^{2}}{\omega_{0}^{2}-\omega^{2}}\left(S+\frac{h}{k} \dot{S}\right)=0 \text { for } x=x_{0} .
\end{aligned}
$$

The latest equation should be coupled with the second equation of (17).
3. Model of the solution of the problem

$(1),(2),(3)$

3.1. Designing of the equation for the problem's own values (9), (10), (11)

The general solution (1) assumes the form

$$
\begin{aligned}
& X(x)=P \cos \lambda x+Q \sin \lambda x+R \cosh \lambda x+S \sinh \lambda x \\
& +\frac{1}{2 \lambda^{3}}\left(\frac{k}{\omega_{0}^{2}-\omega^{2}}+m_{0}\right) \frac{\omega^{2}}{E I} X\left(x_{0}\right)\left[\sinh \lambda\left(x-x_{0}\right)\right. \\
& \left.-\sin \lambda\left(x-x_{0}\right)\right] H\left(x-x_{0}\right)
\end{aligned}
$$

and its second derivative

$$
\begin{gathered}
\ddot{X}(x)=\lambda^{2} P \cos \lambda x+Q \sin \lambda x+R \cosh \lambda x+S \sinh \lambda x \\
+\frac{1}{2 \lambda^{3}}\left(\frac{k}{\omega_{0}^{2}-\omega^{2}}+m_{0}\right) \frac{\omega^{2}}{E I} X\left(x_{0}\right)\left[\sinh \lambda\left(x-x_{0}\right)\right. \\
\left.-\sin \lambda\left(x-x_{0}\right)\right] H\left(x-x_{0}\right) .
\end{gathered}
$$

From (32), (33) and (11) as well as from the values of the function $X(x)$ (additional condition) in $x_{0}$ we obtain the system of equations

$\mathcal{A}(\lambda) V=0$,

where $V^{\mathrm{T}}=\left[Q, S, X\left(x_{0}\right)\right], P=R=0, \omega^{2}=\frac{E I \lambda^{4}}{\rho F}, \mathcal{A}(\lambda)=$

$\left[\begin{array}{ccc}\sin \lambda l & \sinh \lambda l & J\left[\sinh \lambda\left(l-x_{0}\right)-\sin \lambda\left(l-x_{0}\right)\right] \\ -\lambda^{2} \sin \lambda l & \lambda^{2} \sinh \lambda l & J\left[\sinh \lambda\left(l-x_{0}\right)+\sin \lambda\left(l-x_{0}\right)\right] \\ \sin \lambda x_{0} & \sinh \lambda x_{0} & -1\end{array}\right]$, $J=\frac{1}{2 \lambda^{3}}\left(\frac{k}{\omega_{0}^{2}-\omega^{2}}+m_{0}\right) \frac{\omega^{2}}{E I}$. The system (34) has a non-zero solution if

$$
\operatorname{det} \mathcal{A}(\lambda)=0 \text {. }
$$

Zero points of (35) are the characteristic values of the problem (9), (10), (11). These values constitute an infinite sequence of positive elements [2]

$$
\left\{\lambda_{n}\right\} \text {. }
$$

The form of matrix $\mathcal{A}(\lambda)$ shows that $\omega_{0} \neq \omega$.

\subsection{The condition of orthogonality}

The condition of orthogonality of the function space composed from the solutions of the equation

$$
X_{n}^{I V}-\lambda_{n}^{4} X_{n}=\left(\frac{k}{\omega_{0}^{2}-\omega^{2}}+m_{0}\right) \frac{\omega_{n}^{2}}{E I} X_{b}\left(x_{0}\right) \delta_{x_{0}}
$$

and the boundary conditions

$$
X_{n}(0)=X_{n}(l)=0, \quad X_{n}^{I I}(0)=X_{n}^{I I}(l)=0 .
$$

The forms of (37) and (38) are obtained from (9), (11) and (36).

The condition of orthogonality of the solutions of (37) and (38) assumes the form

$$
\begin{aligned}
\int_{0}^{l} & X_{i} X_{j} \mathrm{~d} x \\
+ & \frac{E I}{\rho F}\left[\frac{k(\rho F)^{2} \omega_{0}^{2}}{\left(m_{0} \rho F-E I \lambda_{i}^{4}\right)\left(m_{0} \rho F-E I \lambda_{j}^{4}\right)}+m_{0}\right] \\
& =0 \quad \text { for } i \neq j,
\end{aligned}
$$




$$
\begin{aligned}
& \int_{0}^{l} X_{n}^{2} \mathrm{~d} x+\frac{E I}{\rho F}\left[\frac{k(\rho F)^{2} \omega_{0}^{2}}{\left(m_{0} \rho F-E I \lambda_{n}^{4}\right)^{2}}+m_{0}\right] \\
& =\chi_{n n}>0,
\end{aligned}
$$

obtained in a standard way.

The solution of the problem $(1),(2),(3)$ is a series [4]

$$
\begin{aligned}
& U(x, t)=\sum_{n=1}^{\infty} X_{n}(x) K_{n}(t), \\
& y(t)=\sum_{n=1}^{\infty} A_{n} K_{n}(t),
\end{aligned}
$$

for the cases of (3) and (4), while for (6)

$$
\begin{aligned}
& U(x, t)=\sum_{n=\infty}^{\infty} X_{n}(x) L_{n}(t) \quad \text { for } \quad x \neq x_{0}, \\
& U\left(x_{0}, t\right)=\sum_{n=1}^{\infty} X_{n}\left(x_{0}\right) M_{n}(t) \text { for } x=x_{0}, \\
& y(t)=\sum_{n=1}^{\infty} A_{n} N_{n}(t),
\end{aligned}
$$

while functions $K_{n}(t)$ in (40) and (41) are solutions of (12) (case (3)) or (14) (case (4)) respectively, completed with the right sides obtained from $f_{1}(x, t)$ or $f_{2}(x, t)$ issuing from the distributed in a series $X_{n}(x)$ as the space base.

In (42), (43) and (44) the computations are analogical to those for (40) and (41).

\section{Conclusion}

The obtained results may be used in the construction of damping systems, controlling systems and those minimizing the vibrations transferred from the base to the object.
Final conclusions:

1. The hitherto prevailing view regarding the separation of variables in the linear discrete-continuous systems has been proved wrong. It was not assumed that the specific problems of those systems are identical for all parameters of the structure, that is, the parameters determining elasticity and internal damping.

2. The way of separation of variables presented in the work indicates that the method can be applied for more complex systems.

3. The local structure of the beam in the surroundings of the point of application of the force depends in a significant way on the parameters of the structure of the system.

4. The presented computational model is the basis for very interesting special cases.

\section{References}

[1] P. Antosik, J. Mikulski, R. Sikorski, Theory of Distributions; the Sequential Approach, Elsevier Sci. Publ., New York 1973.

[2] F.P. Beer, E.R. Johnson, (Jr.), Mechanics for Engineers, Cliffs, New Yersey 1977.

[3] S. Kasprzyk, S. Sędziwy, Bull. Acad. Sci. Math. 31, 329 (1983).

[4] S. Kasprzyk, S. Sędziwy, Bull. Acad. Sci. Tech. Sci. 43, 413 (1995).

[5] L. Schwartz, Mathematics for Physical Sciences, Dover Books on Mathematics, Dover Publ., Dover 2008. 